\title{
Behavior of reinforced concrete beams pre-cracked and repaired by composite materials under static loading
}

\author{
Mostefa Hamrat ${ }^{1}$, Bensaid Boulekbache ${ }^{1}$, Halima Bouziane ${ }^{1}$ and Hayet Benkara ${ }^{1}$ \\ ${ }^{1}$ Département de Génie Civil, Université Hassiba Benbouali, Chlef, Algérie
}

\begin{abstract}
This work constitutes a contribution to the analysis of the behavior of beams repaired by composite materials. To analyze the overall behavior and failure modes of the beams, an experimental study of nine reinforced concrete beams, pre-cracked and then repaired by composite materials was conducted. Six beams were pre-cracked and repaired in the tensioned part (bending repair) and in the other two beams on the tensioned and lateral parts with strips in the shape of $U$ (shear repair). A comparative study was made between the ultimate moments measured experimentally and those calculated by the theoretical models. Compared to the control beam, the resistance gain for the beams repaired in bending is $50 \%$ to $90 \%$, while that of beams repaired in shear is from $120 \%$ to $177 \%$. The beams repaired in shear exhibit a ductile rupture in bending. However, the beams repaired in bending were failed by the lift-off of composite or by failure of concrete cover layer (except for beams repaired by fiber glass). BAEL99, EC2-04 and ACI318-08 models give the best prediction of the ultimate moments with a mean value of 1.16 for the ratio of $\mathrm{M}_{\mathrm{Exp}} \cdot / \mathrm{M}_{\text {theor }}$. and a mean standard deviation of 0.33 .
\end{abstract}

\section{Introduction}

La durée de vie des ouvrages en béton armé est de l'ordre de 50-100 ans, mais elle se trouve écourtée du fait de nombreuses pathologies. Un grand nombre d'ouvrages présentent un état de dégradation avancée causé par une exposition prolongée à un environnement agressif, des défauts de résistance à l'effort tranchant ou en flexion due au chargement excessif, ainsi que celle liée à la fatigue de la structure sous chargement cyclique sont à l'origine de la diminution de la durée de vie de ces ouvrages. Face à ces problèmes, la réhabilitation et le renforcement des ouvrages, deviennent un enjeu stratégique.

La réparation des ouvrages en béton armé représente un moyen avantageux d'améliorer leur capacité portante et de prolonger leur durée de vie. En effet, il est souvent beaucoup moins onéreux de réparer certains éléments structuraux que d'effectuer une reconstruction complète de l'ouvrage. Plusieurs techniques de réparation sont disponibles sur le marché, dont celle qui consiste dans la réparation externe avec des matériaux composites. Face à cette situation, il est nécessaire de posséder un certain nombre de connaissances sur le comportement chargeflèche, ductilité et mode de rupture des éléments structuraux en béton armé réparées par des matériaux composites.

Par ailleurs, l'apport des matériaux composites en tant que matériau de réparation soulève de nouvelles questions. Afin d'obtenir une utilisation adéquate de ces nouveaux matériaux dans les tâches de réhabilitation et de renforcement, il est nécessaire de bien comprendre leur interaction avec le béton armé. Toutefois, des modèles ont été formulées par des groupes de travail tel qu'on les trouve dans l'approche de la Fédération Internationale du Béton (FIB) [1] et de l'Association Française de Génie Civil (AFGC) [2], à partir des résultats de la littérature. Ces modèles ont besoin d'être expérimenté et calibré.

\section{Programme expérimental}

Lors de cette étude, la valeur de la résistance souhaitée du béton a été prise égale à $25 \mathrm{MPa}$, valeur courante et proche du chantier.

Neuf spécimens de poutres de section $0,10 \times 0,15 \mathrm{~m}^{2}$ et de $1.10 \mathrm{~m}$ de portée, ont été fabriqués et testés (figure 1). Ces poutres sont armées par deux barres HA8 dans la partie tendue et par deux barres HA6 dans la partie comprimée, ce qui donne une section sous-armée.

Les huit spécimens d'épreuve endommagés jusqu'à un niveau de chargement correspondant à une ouverture de fissure de 0,3 $\mathrm{mm}$ [3] (figure 2), vont être réparées, en utilisant le collage des lamelles composite (carbodur) et des tissus (fibre de carbone et fibre de verre). 


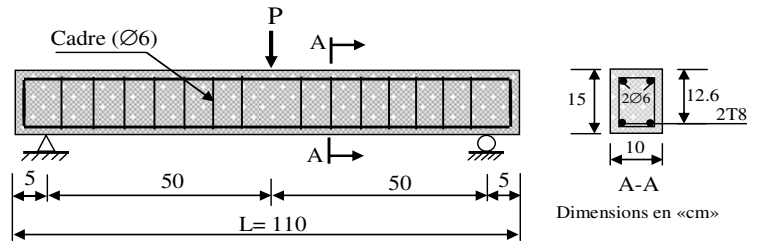

Fig. 1. Dimensions et armatures des poutres.

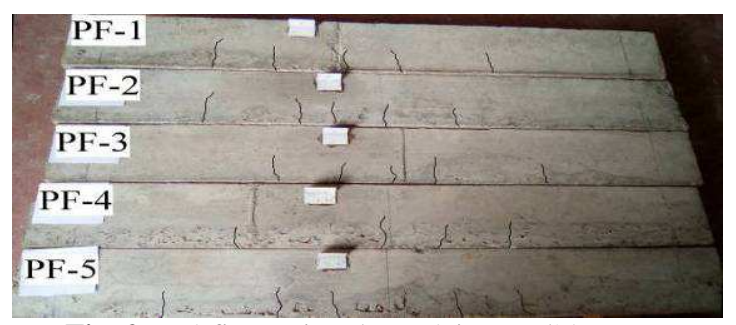

Fig. 2. Pré-fissuration des spécimens d'épreuve.

Deux modes de réparation sont utilisées dans notre étude. Dans le premier mode (A): le composite est collé sur la face tendue avec une largeur égale à celle de la poutre et une distance de $30 \mathrm{~mm}$ entre l'extrémité du tissu (lamelle) et l'appui de la poutre. Pour le deuxième mode (B), le composite est collé sur les faces tendue et latérales en forme de $\mathrm{U}$ (tableau 1).

Tableau 1. Configuration des Poutres Testées

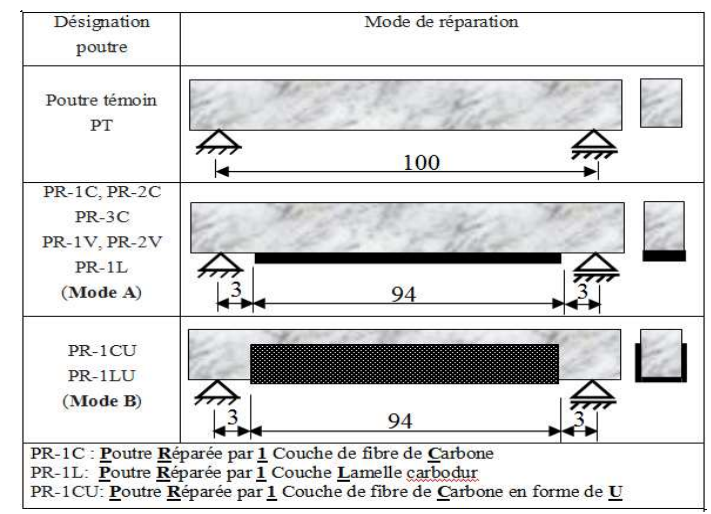

\section{Résultats et discussions}

\subsection{Réponse charge-flèche des poutres réparées}

La résistance ultime des poutres réparées est principalement dictée par le mode de réparation.

\subsubsection{Mode de réparation $A$}

Dans cette série, on a utilisé les fibres (les fibres de carbone unidirectionnelles, les fibres de verre bidirectionnelles et des lamelles carbodur) en 1,2 et 3 couches. Ces couches sont collées dans la face tendue de la poutre (réparation en flexion).

La figure 3 représente les courbes charge-flèche de la poutre témoin et des poutres réparées selon le mode de réparation $\mathrm{A}$.
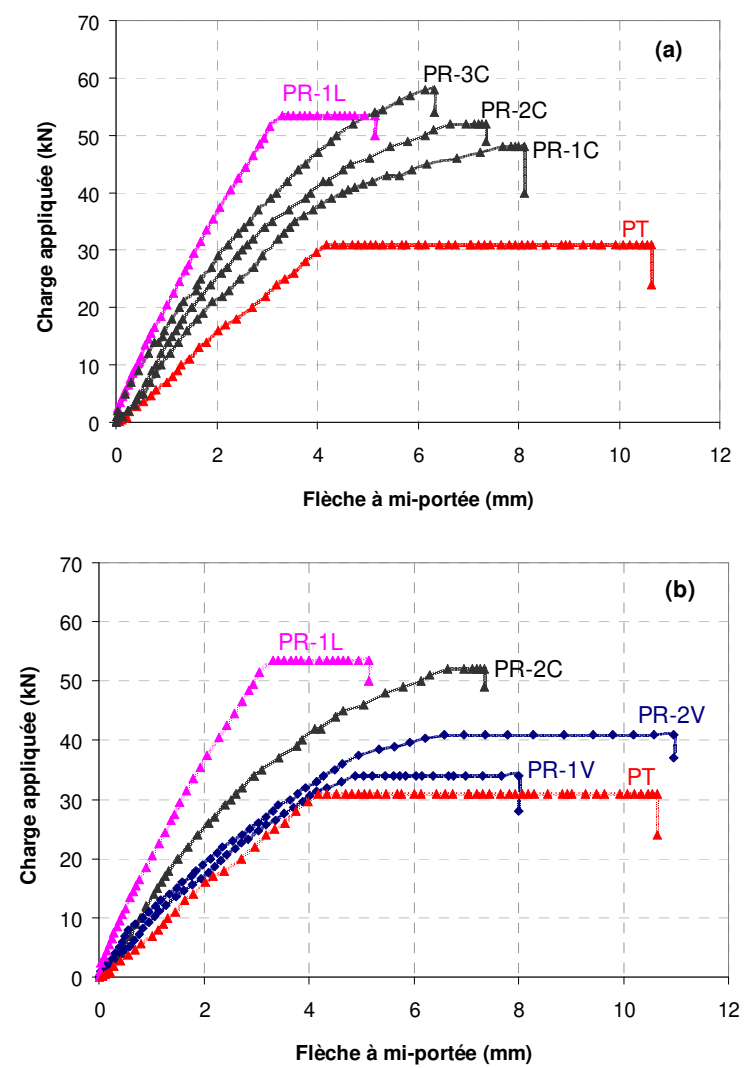

Fig. 3. Courbes charge-flèche des poutres réparées (mode A)

A l'issue de la figure 3, on observe ce qui suit:

-De grandes différences de comportement apparaissent entre la poutre témoin (PT) et les poutres réparées: PR-1C, PR-2C et PR-3C. (figure 3(a)). Les poutres réparées possèdent un pseudo-palier plastique de ductilité une fois la résistance ultime atteinte.

-Les poutres PR présentent un comportement plus rigide avec une réduction significative de la flèche (figure 3(a)). Dans ce sens, les capacités portantes des poutres réparées sont: $48 \mathrm{kN}$ pour $\mathrm{PR}-1 \mathrm{C}, 52 \mathrm{kN}$ pour $\mathrm{PR}-2 \mathrm{C}$ et $58 \mathrm{kN}$ pour $\mathrm{PR}-3 \mathrm{C}$, ce qui représente des gains par rapport à la poutre témoin respectivement de: $55 \%, 70 \%$ et $90 \%$ (tableau 2). Les flèches correspondant à ces charges sont de $8,0 \mathrm{~mm}, 7,3 \mathrm{~mm}$ et $6,3 \mathrm{~mm}$ alors qu'elle était de $10,6 \mathrm{~mm}$ pour la poutre témoin.

-La charge de rupture de la poutre PR-2V est de $78 \%$ la charge de rupture de la poutre PR-2C. Cependant, la poutre $\mathrm{PR}-2 \mathrm{~V}$ présentait une flèche de $11 \mathrm{~mm}$ à la rupture où la poutre PR-2C s'était avec une flèche de $7,4 \mathrm{~mm}$ (figure 3(b)), ceci est principalement lié aux propriétés de la fibre de verre $(4,8 \%$ élongation à la rupture contre 1,8 $\%$ pour la fibre de carbone) [4].

-L'ajout des lamelles à base de fibres de carbone augmente la charge de rupture et réduit la flèche (figure 3(b)). En effet, la poutre PR-1L a enregistré un gain de résistance de $87 \%$ par rapport à la poutre témoin. De plus, lamelle carbodur augmente d'une manière substantielle la rigidité de la poutre. La poutre PR-1L présentait une charge de rupture presque égale à la charge de rupture de la poutre PR-2C ( 1 Plaque carbodur $\approx 2$ couches de fibres de carbone).

-Les courbes des poutres PR montrent bien le point de plastification qui se situe aux environs de $50 \mathrm{kN}$. Pour 
des flèches semblables qui varient de 4 à $6 \mathrm{~mm}$, ceci représente des gains de $40 \%$ environ par rapport à la poutre témoin. C'est donc dire que l'utilisation des matériaux composites en flexion permet de retarder la plastification des armatures longitudinales tendues.

Tableau 2. Résultats expérimentaux à la rupture

\begin{tabular}{|c|c|c|c|c|c|}
\hline Poutre & $\begin{array}{c}\mathrm{P}_{\mathrm{u}} \\
\mathrm{KN}\end{array}$ & $\begin{array}{c}\Delta_{\mathrm{u}} \\
\mathrm{mm}\end{array}$ & $\begin{array}{c}\text { Augm } \uparrow \\
\text { Charge } \\
\%\end{array}$ & $\begin{array}{c}\text { Dimin } \downarrow \\
\text { flèche } \\
\%\end{array}$ & $\begin{array}{c}\text { Mode de } \\
\text { Rupture }\end{array}$ \\
\hline PT & 31 & 10,6 & - & - & Flexion \\
\hline PR-1C & 48 & 8,1 & 55 & 24 & $\begin{array}{c}\text { Décollement } \\
\text { du } \\
\text { composite }\end{array}$ \\
\hline PR-3C & 58 & 6,3 & 90 & 41 & $\begin{array}{c}\text { Rupture } \\
\text { enrobage du } \\
\text { béton }\end{array}$ \\
\hline PR-1V & 34 & 8,0 & 10 & 38 & $\begin{array}{c}\text { Rupture } \\
\text { enrobage du } \\
\text { béton }\end{array}$ \\
\hline PR-2V & 40,5 & 11,0 & 31 & $4{ }^{*}$ & $\begin{array}{c}\text { Flexion } \\
\text { Flexion }\end{array}$ \\
\hline PR-1L & 54 & 5,2 & 87 & 46 & $\begin{array}{c}\text { Rupture } \\
\text { enrobage du } \\
\text { béton }\end{array}$ \\
\hline PR-1CU & 68 & 9,5 & 120 & 12 & $\begin{array}{c}\text { bris de fibre } \\
\text { de carbone }\end{array}$ \\
\hline PR-1LU & 86 & 12,1 & 177 & $14^{*}$ & $\begin{array}{c}\text { Décollement } \\
\text { Cassure de } \\
\text { la plaque }\end{array}$ \\
\hline$*$ A & 56 & 70 & 30 &
\end{tabular}

Augmentation

\subsubsection{Mode de réparation $B$}

Cette série comprend deux poutres réparées par tissu de carbone/lamelles en forme de U. Cet essai va nous permettre de comparer le mode de réparation $\mathrm{A}$, avec le mode de réparation $B$ (configuration en $\mathrm{U}$ ).

La figure 4 représente les courbes charge-flèche des poutres selon le mode de réparation $B$ et la poutre témoin.

D'après ces courbes, on observe ce qui suit:

- La réparation d'une poutre augmente d'une manière substantielle la charge de rupture, en améliorant la rigidité et en réduisant la flèche. On note que la poutre PR-1CU a permis d'atteindre une augmentation de charge significative de près de $120 \%$ par rapport à la poutre témoin (tableau 2). De plus, cette poutre réparée présentait une flèche de $9,5 \mathrm{~mm}$ seulement au niveau de charge où la poutre témoin s'était avec une flèche de 10,6 $\mathrm{mm}$ (figure 4).

- Pour le mode de réparation B, les courbes chargeflèche ont présenté une très grande rigidité par rapport aux poutres réparées selon mode de réparation $\mathrm{A}$. Les charges de ruptures sont: $68 \mathrm{kN}$ pour PR-1CU et $86 \mathrm{kN}$ pour PR-1LU, ce qui représente des gains par rapport aux poutres réparées uniquement en flexion (PR-1C et PR1L) de $42 \%$ et $59 \%$, respectivement (Tableau 2).

En outre, il faut noter que ces deux poutres réparées en cisaillement par des bandes continues en $U$ sur toute leur longueur aient permis d'atteindre une rupture ductile en flexion (figure 8).

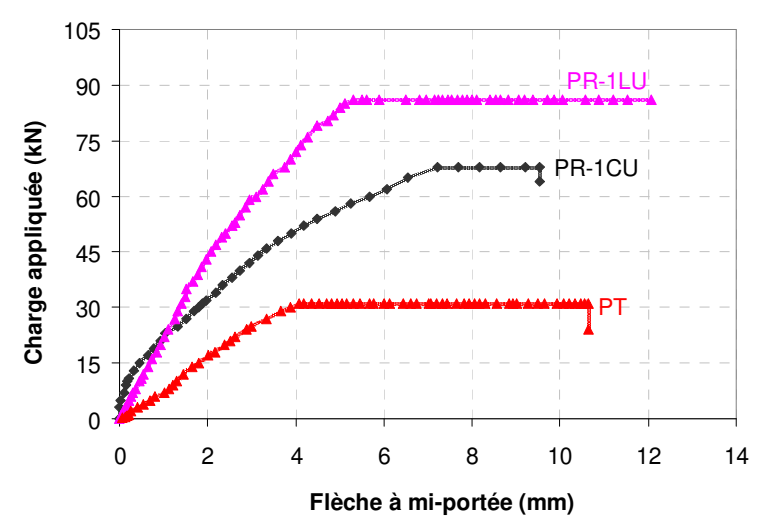

Fig. 4. Courbes charge-flèche des poutres réparées (mode B)

\subsection{Faciès et modes de rupture des poutres}

La combinaison des matériaux composites comme réparation de poutres en béton armé produit de nouveaux modes de rupture. D'après Meier [5], ces ruptures peuvent se situer à différents niveaux: dans la poutre en béton armé, dans le composite ou dans l'interface bétoncomposite.

Selon les modes de réparation, les principaux modes de rupture susceptibles d'être observés dans les poutres réparées sont les suivants:

\subsubsection{Mode de réparation A}

Poutre: PR-1C.

La rupture de la poutre est due au décollement partiel du composite. Ce décollement de composite qui débute aux extrémités de celui-ci, puis se propage vers le centre de la poutre. Le décollement s'est produit entre le béton et l'adhésif du composite (figure 5). Ce mode de rupture provient de la concentration de contraintes à l'extrémité du tissu.
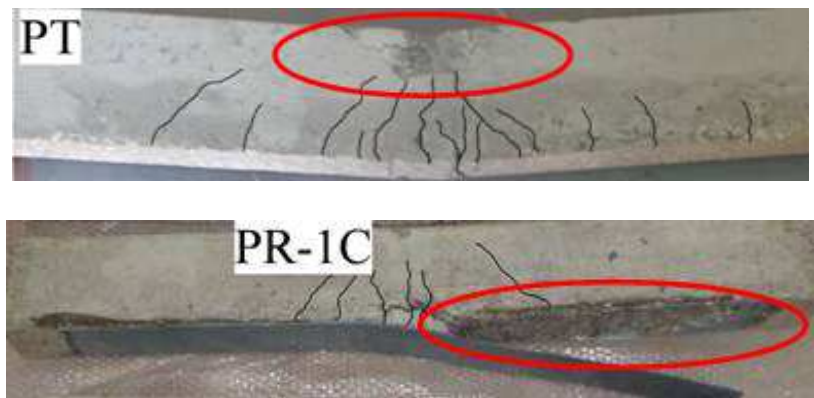

Fig. 5. Mode de rupture des poutres (PT + PR-1C).

Poutres: PR-2C + PR-3C + PR-1L

Il y a eu apparition de fissures de flexion sur la zone centrale, dès le début du chargement. Des fissures verticales apparaissent à l'extrémité où le moment fléchissant est maximal, et sont inclinées vers le vers le point d'application de la charge (figure 6).

La rupture de ces poutres a été fragile et soudaine et s'est caractérisée par décollement du composite avec une séparation complète de l'enrobage du béton sous une fissure diagonale d'effort tranchant (figure 6). Certes, le 
décollement du composite s'est produit entre le béton et la colle. En effet, la résistance à la contrainte de cisaillement de la colle est plus élevée que celle du béton.
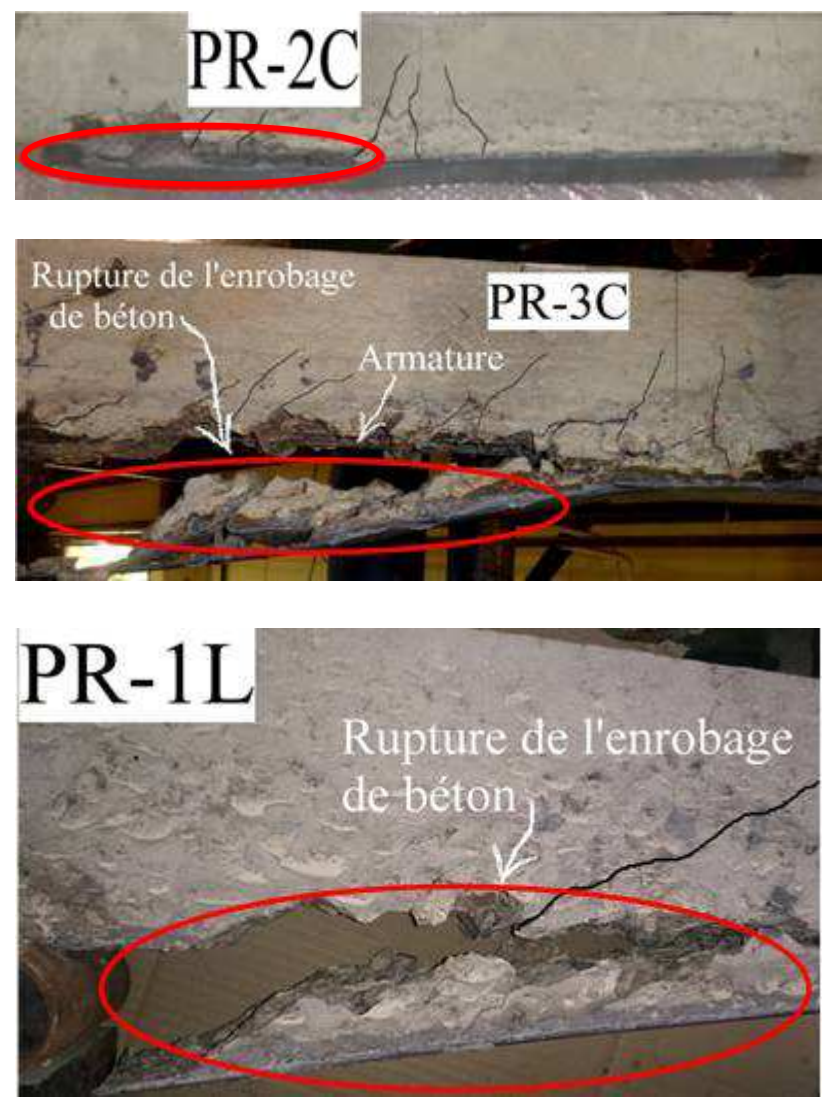

Fig. 6. Mode de rupture des poutres (PR-2C+ PR-3C+ PR-1L).

\section{Poutres: PR-1V + PR-2V}

Des fissures de flexion se forment au niveau de la zone tendue et dans la partie centrale de la poutre telle qu'en figure 7. A des niveaux de chargement relativement élevés, des petites fissures diagonales se développent de part et d'autre de la zone centrale de la poutre. La poutre s'est rompue en flexion où la zone comprimée s'est écrasée avec rupture du composite (figure 7).
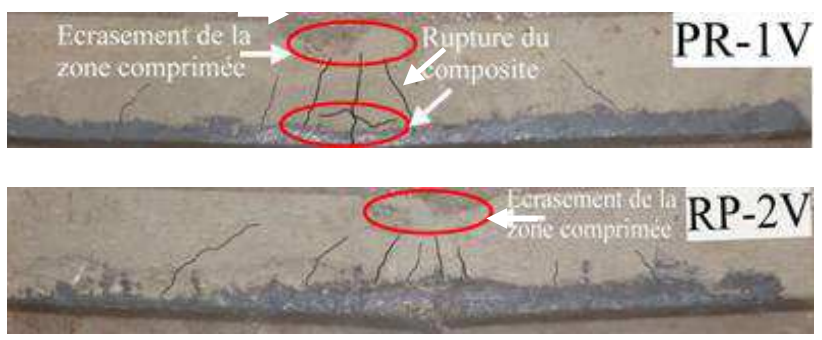

Fig. 7. Mode de rupture des poutres (PR-1V+ PR-2V).

\subsubsection{Mode de réparation $B$}

\section{Poutre: PR-1CU}

Un bris de fibre de carbone a été observé dans la face tendue et les deux faces latérales. Il y a eu apparition de fissures diagonales sur la face latérale de la poutre (figure
8). La réparation de la poutre par collage du tissu continu en forme de $U$ ne permet pas de "coudre" les fissures. Dans le Mode de réparation B, le composite est sollicité jusqu'à la rupture et que dans le Mode de réparation A, on a assisté au décollement du composite ou rupture de l'enrobage de béton.

La séquence de rupture était moins brutale. En fait, cette poutre présente plus de fissures (durant la phase de préfissuration). Ces fissures libèrent l'énergie stockée graduellement de sorte que, à l'état ultime, la quantité finale d'énergie libérée ne soit pas aussi grande, et par conséquent la rupture est moins explosive et suivi d'un bruit assez distinct et particulier dû aux craquements du composite.

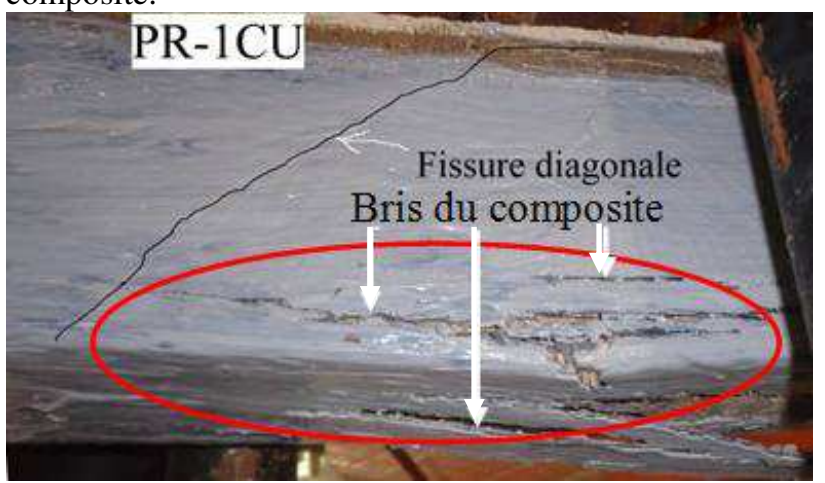

Fig. 8. Rupture de la poutre PR-1CU.

\section{Poutre: PR-1LU}

La poutre a été rompue par décollement et cassure de la lamelle collée dans la face latérale sous une sévère fissure diagonale d'effort tranchant à l'extrémité de la plaque. Cette rupture est causée par un déplacement relatif vertical de la face fissurée (figure 9). L'étrier en U fait de lamelles carbodur a recélé toutes fissures de flexion.

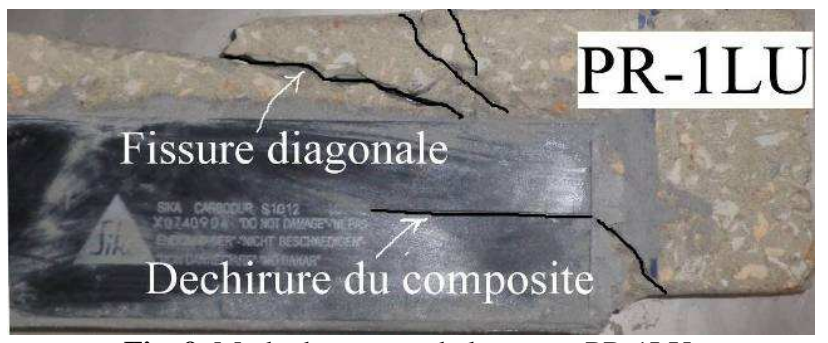

Fig. 9. Mode de rupture de la poutre PR-1LU.

\subsection{Ductilité des poutres testées}

Si la rigidité, la charge et la flèche conditionnent le comportement structurel avant le pic de chargement [6],[7], c'est uniquement la ductilité qui a une influence importante sur le comportement post-pic [8]. Certes, la ductilité d'un élément représente sa capacité à se déformer après la phase élastique. Par souci de simplification, les règlements préfèrent utiliser la notion de déplacement, pour définir l'indice de ductilité comme étant le rapport du déplacement ultime $\left(\Delta_{\mathrm{u}}\right)$ sur le déplacement limite du comportement élastique $\left(\Delta_{\mathrm{y}}\right)$. L'indice de ductilité est défini comme suit : 


$$
\mu_{\Delta}=\frac{\Delta_{u}}{\Delta_{y}}
$$

Les valeurs de l'indice de ductilité $\left(\mu_{\Delta}\right)$ sont représentées sur la figure 10 .

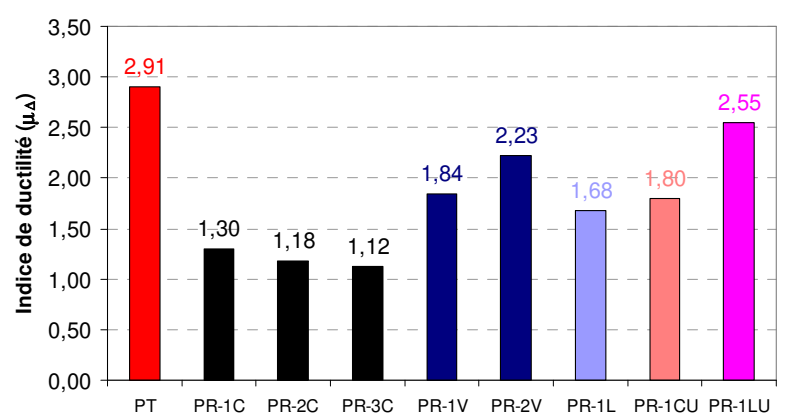

Fig.10. Indices de ductilité des poutres testées.

A partir de la figure 10, on constate que les valeurs de l'indice de ductilité des poutres réparées en cisaillement sont supérieures aux valeurs des poutres réparées en flexion, à l'exception pour les poutres réparées par la fibre de verre où l'indice de ductilité est de 1,84 pour PR$1 \mathrm{~V}$ et 2,23 pour $\mathrm{PR}-2 \mathrm{~V}$. Ceci prouve que la réparation par plaque carbodur en forme $\mathrm{U}$ rend la poutre ductile. Par contre, la réparation en flexion mène à une rupture prématurée, et donc à une rupture fragile (indice de ductilité faible)

\subsection{Validation des méthodes de dimensionnement}

Le dimensionnement d'une poutre réparée par des matériaux composites à l'aide des méthodes analytiques comprend le calcul du moment fléchissant ultime. Le calcul du moment ultime repose sur les hypothèses conventionnelles du béton armé en tenant compte du comportement élastique linéaire du composite. Ces méthodes sont utilisées sans coefficient de sécurité, afin de comparer les résultats obtenus aux résultats expérimentaux.

En utilisant le modèle de calcul à l'ELU de la méthode FIB [1], nous estimons la résistance ultime au moment fléchissant d'une poutre réparée par des matériaux composites.

Etant donnée la section transversale d'une poutre réparée (figure 11).

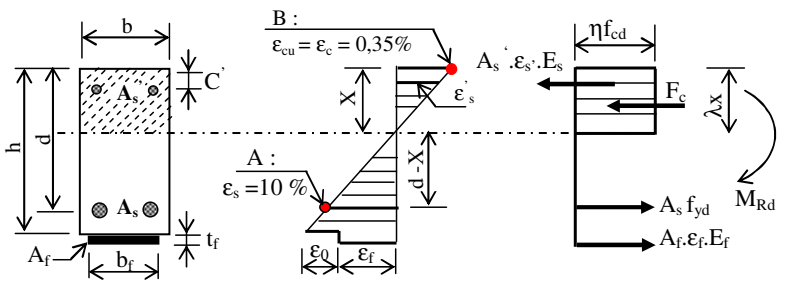
(a). Géométrie
(b). Déformation
(c). Contrainte

Fig.11. Section transversale d'une poutre réparée.

Le moment fléchissant résistant $\left(\mathrm{M}_{\mathrm{R}}\right)$ est calculée au centre de poutre par:
$M_{R}=A_{s} \cdot f_{y d}\left(d-\frac{\lambda \cdot x}{2}\right)+A_{f} \cdot E_{f} \cdot \mathcal{E}_{f}\left(h-\frac{\lambda \cdot x}{2}\right)$

$\varepsilon_{f}=\frac{h-x}{x} \varepsilon_{c u}$ (déformation du composite)

Les symboles définis pour expliquer les méthodes de base des 3 règlements sont utilisés (figure 12).

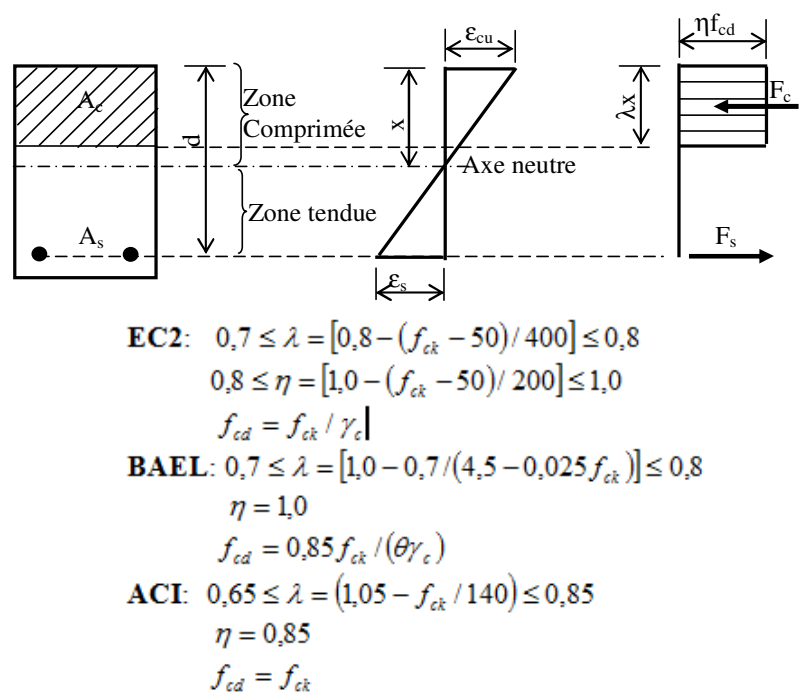

Fig.12. Diagramme de déformation et diagramme simplifié des forces équivalentes (valeurs $\lambda$, $\eta$ et $\mathrm{f}_{\mathrm{cd}}$ pour l'EC2, le BAEL et l'ACI318).

Le tableau 3 donne les valeurs du Moment fléchissant résistant prédites par les 3 règlements (BAEL [9], EC2 [3] et ACI318 [10]).

Tableau 3. Moment fléchissant résistant.

\begin{tabular}{|c|c|c|c|c|c|c|c|}
\hline \multirow{2}{*}{ Poutre } & $\mathrm{M}_{\exp }$ & \multicolumn{3}{|c|}{ Moment calculé } & \multicolumn{3}{|c|}{$\mathrm{M}_{\text {exp }}$. $\mathrm{M}_{\text {cal. }}$} \\
\cline { 3 - 8 } & & BAEL & EC2 & ACI & BAEL & EC2 & ACI \\
\hline PR-1C & 12,1 & 7,5 & 7,5 & 6,7 & 1,61 & 1,60 & 1,81 \\
\hline PR-2C & 13,1 & 11,2 & 11,3 & 9,7 & 1,17 & 1,16 & 1,35 \\
\hline PR-3C & 14,6 & 15,6 & 18,9 & 15,8 & 0,94 & 0,77 & 0,92 \\
\hline PR-1V & 8,6 & 6,4 & 6,5 & 5,8 & 1,35 & 1,33 & 1,47 \\
\hline PR-3V & 10,2 & 9,3 & 9,5 & 8,3 & 1,10 & 1,08 & 1,23 \\
\hline PR-1L & 13,6 & 22,2 & 22,4 & 18,5 & 0,61 & 0,61 & 0,73 \\
\hline \multicolumn{3}{|c|}{ Moyenne } & $\mathbf{1 , 1 3}$ & $\mathbf{1 , 0 9}$ & $\mathbf{1 , 2 5}$ \\
\hline \multicolumn{4}{|c|}{ Ecart type } & $\mathbf{0 , 3 1}$ & $\mathbf{0 , 3 3}$ & $\mathbf{0 , 3 5}$ \\
\hline \multicolumn{4}{|c|}{ Coef de variation (\%) } & $\mathbf{2 7}$ & $\mathbf{3 0}$ & $\mathbf{2 8}$ \\
\hline
\end{tabular}

A l'issue du tableau 3, il est clair que la variation du moment fléchissant entre les valeurs expérimentales et les valeurs prédites par les trois règlements est très faible, avec un coefficient de variation de $28 \%$. Cependant, les trois règlements surestiment le moment fléchissant pour la poutre PR-1L, ceci est principalement lié aux propriétés du composite (module d'élasticité du composite (lamelle carbodur + résine) $\left(\mathrm{E}_{\mathrm{f}}\right)$ et son épaisseur). En outre, le mode de rupture de cette poutre réparée en flexion est provoqué par flexion et par l'effort tranchant. 


\section{Conclusions}

Les conclusions tirées de cette étude sont les suivantes:

- Les poutres réparées en flexion ont obtenu des gains de résistance, allant de $50 \%$ à $90 \%$, par rapport à celle de la poutre témoin, et une diminution de la flèche variant de 25 à $45 \%$.

- Les poutres réparées: RP-1V et RP-2V ont obtenu des gains de résistance par rapport à la poutre témoin respectivement de $10 \%$ et $31 \%$. Cependant, ces poutres possèdent un pseudo-palier plastique de ductilité une fois la résistance ultime atteinte. En effet, l'indice de ductilité était de 1,84 pour la poutre RP-1V et 2,23 pour la poutre RP-2V.

- L'ajout d'une plaque carbodur sur la face tendue de la poutre (PR-1L) contribue à augmenter la rigidité de la poutre et à diminuer la flèche. Cependant, on note un gain de charge à la rupture de $87 \%$ par rapport à la poutre de référence.

- L'utilisation d'étriers de fibres de carbone et lamelle carbodur en forme de $\mathrm{U}$ permet de réparer et rigidifier la poutre. Un gain de résistance significatif de $120 \%$ a été enregistré pour la poutre PR-1CU, alors qu'un gain était de $177 \%$ pour la poutre PR-1LU. Par ailleurs, l'indice de ductilité était de 2,55 pour la poutre $\mathrm{PR}-1 \mathrm{LU}$ et 1,80 pour la poutre $\mathrm{PR}-1 \mathrm{CU}$.

- Les poutres réparées en flexion ont été rompues par décollement du composite ou rupture de l'enrobage de béton, à l'exception pour les poutres réparées par la fibre de verre, ces deux poutres (PR-1V et PR-2V) aient permis d'atteindre une rupture ductile en flexion.

- Les trois règlements (BAEL99, EC2-04 et ACI318-08) sont acceptables vis-à-vis du moment résistant ultime des poutres réparées par des matériaux composites, avec un coefficient de variation de $28 \%$.

\section{Références}

1. FIB, Externally bonded FRP reinforcement for RC structures. Technical report, Bulletin 14, ISBN 288394-055-X, (2001).

2. AFGC, Réparation et renforcement des structures en béton au moyen des matériaux composites, Recommandations provisoires, (2003).

3. Eurocode 2, Calcul des structures en béton -Partie 11: Règles générales et règles pour les bâtiments, ENV 1992-1-1, (2004).

4. Guide des Notices Techniques, web:dza.sika.com, Sika El Djazair.

5. MEIER, Carbon Fiber-Reinforced Polymers: Modem Materials in Bridge Engineering, Structural Engineering International, $\mathrm{n}^{\circ} .1$, (1992), pp.7- 12.

6. M. Hamrat, Comportement structural du béton à hautes performances (flexion et effort tranchant),
Thèse de doctorat, Université USTHBAlgérie/Université européenne de Bretagne-France, (2010).

7. M. Hamrat, B. Boulekbache, M. Chemrouk. S. Amziane, Flexural cracking behavior of normal strength, high strength and high strength fiber concrete beams, using Digital Image Correlation technique, Construction and Building Materials 106, (2016), pp. 678-692.

8. M. Hamrat, B. Boulekbache, M. Chemrouk. S. Amziane, Flexural, Behavior of reinforced concrete beams: Comparative analysis between highperformance concrete and ordinary concrete, MATEC Web of Conferences 11, (2014), 02003.

9. BAEL, règle technique de conception et de calcul des ouvrages et constructions en béton armé suivant la méthode des états limites, (1999).

10. ACI Committee 318, Building Code Requirements for Structural Concrete (ACI 318-08) and Commentary (318R-05), American Concrete Institute, Farmington Hills, MI, (2008), 430 p. 